\title{
Immunoalkaline phosphatase technique applied to paraffin wax embedded tissues in diagnostic renal pathology
}

\author{
R Jackson, E R Holme, G M Phimister, A Kennedy, A L C McLay
}

\begin{abstract}
An indirect immunoalkaline phosphatase (IAP) technique was used to evaluate the glomerular deposition of immunoglobulins, C3, C1q and fibrinogen. In 80 renal biopsy specimens the results obtained using this technique were compared with those obtained by direct immunofluorescence to see if it could be used as a viable alternative. The IAP technique was straightforward to perform, it yielded quick results, and was highly reproducible, provided that a standardised short fixation period of two and a half hours was used. For the detection of immunoglobulin deposits, the IAP results correlated well with those of immunofluorescence. Despite poorer performance in identifying complement components and fibrinogen it could, within certain limits, provide an adequate diagnostic alternative to immunofluorescence. Each technique gave false negative results, those of immunofluorescence being related to its failure to identify mesangial deposition of IgA in two cases where its distribution seemed to be focal, and those of IAP to a failure to detect linear deposition of IgG in all three cases of anti-glomerular basement membrane disease.
\end{abstract}

Direct immunofluorescence on frozen biopsy material has long been regarded as an essential element in the diagnosis of glomerular disease. ${ }^{1}$ More recently, immunoperoxidase methods applied to frozen biopsy material have been proposed as a considerable improvement. ${ }^{2-4}$ After Curran and Gregory observed that antigenic epitopes concealed by fixation can be unmasked by trypsinisation, ${ }^{5}$ immunoperoxidase techniques were shown to be practicable on paraffin wax embedded renal biopsy material..$^{6-8}$ These latter studies were performed retrospectively on material fixed in buffered formalin for periods from six to over 24 hours. Concordance rates between the immunoperoxidase and immunofluorescence methods for all antibodies tested were quoted as $81 \%$ and $79 \%$, respectively. ${ }^{78}$ It was evident that the technique was easy to perform, had the advantage of a permanent preparation, and gave more accurate localisation of immune deposits than immunofluorescence. A lower level of sensitivity, however, was noted for the detection of C3.

Our own aim was to establish an accurate and reproducible technique which would remain reliable despite changing technical staff. Prospective comparative analysis with immunofluorescence would permit assessment of its accuracy and indicate whether it could, in all circumstances, be used as a satisfactory alternative to immunofluorescence. Initial results on retrospectively identified positive cases were disappointing. Despite following published methods we obtained numerous false negative results while other cases were associated with high background staining which made interpretation impossible.

Moreover, there was wide variation from case to case in the degree of trypsinisation required to reproduce the immunofluorescence findings. None the less the advantages of a paraffin wax based technique were clear, particularly where small biopsy size limited the available diagnostic material. As the most clearly identifiable variable in specimen preparation was the wide range of fixation times we felt that this might be the principal reason for inconsistent results. A short fixation period of two and a half hours, which would be likely to limit epitope degradation, was selected and all samples from November 1987 onward were subjected to such a regimen. During the initial period of developing the technique it was noted that an alkaline phosphatase signalling system $^{9}$ consistently gave better results than immunoperoxidase and was therefore adopted for the trial period. Our aim was, in a prospective study, to compare the accuracy and value of a short fixation immunoalkaline phosphatase technique with a standard frozen section immunofluorescence method in the glomerular localisation of immunoglobulins, complement components, and fibrinogen. As the immunofluorescence studies on biopsy material from Glasgow Royal Infirmary were routinely performed at the Immunopathology Department of the Western Infirmary, Glasgow, the information provided by each technique could be readily documented without bias.

\section{Methods}

Between January and November 1988 all biopsy material from the Glasgow Royal Infirmary renal unit underwent an invariable period of two and a half hours buffered formalin fixation before paraffin wax embedding. After fixation tissue was dehydrated and processed through graded alcohols to paraffin wax within a variable cycle time not exceeding 20.5 hours. In addition to conventional staining, spare sections were stained using the indirect 
Table 1 Antibody identity and concentration used for IAP technique

\begin{tabular}{|c|c|c|}
\hline & Manufacturer & Dilution \\
\hline $\begin{array}{l}\text { Rabbit anti-human IgG } \\
\text { Rabbit anti-human IgA } \\
\text { Rabbit anti-human IgM } \\
\text { Rabbit anti-C1q } \\
\text { Rabbit anti-C3 } \\
\text { Rabbit anti-fibrinogen }\end{array}$ & (Dako) & $\begin{array}{l}1 \text { in } 8000 \\
1 \text { in } 8000 \\
1 \text { in } 3000 \\
1 \text { in } 2000 \\
1 \text { in } 4000 \\
1 \text { in } 4000\end{array}$ \\
\hline
\end{tabular}

immunoalkaline phosphatase (IAP) method described below. Unmasking of the complexed antigens was achieved by trypsinisation, the duration of digestion depending on the primary antibody in use. Digestion times were established by serial titrations in known positive cases and were similar to those used by MacIver et $a l .{ }^{67}$

The protocol for the immunoalkaline phosphatase technique was as follows:

1 Cut thin paraffin section ( $3 \mu \mathrm{m})$, mount on uncoated glass slides, and dry at $60^{\circ} \mathrm{C}$ for 30 minutes.

2 Dewax in xylene and hydrate through graded alcohols.

3 Wash in TRIS-saline and warm up in TRIS-buffer at $37^{\circ} \mathrm{C}$ for five minutes.

4 Digest sections in $0.1 \%$ trypsin (Difco) in $0.1 \%$ calcium chloride at $37^{\circ} \mathrm{C}$, for 50 minutes for anti-immunoglobulin antibodies, $30 \mathrm{~min}$ utes for anti-complement components, and 10 minutes for anti-fibrinogen.

5 Immerse slides in cold distilled water for five minutes to stop enzyme activity.

6 Wash three times in TRIS-saline for 10 minutes and apply normal swine serum (Gibco) diluted 1 in 5 with $0.05 \mathrm{M}$ TRIS buffer at $\mathrm{pH}$ $7 \cdot 6$ for 15 minutes.

7 Primary rabbit antibodies which previously have been absorbed with guinea pig and porcine liver acetone powders (Sigma) are applied for 30 minutes in the concentrations listed in table 1 .

8 Wash twice in TRIS-saline for 10 minutes and then apply normal swine serum diluted 1 in 5 with $0.05 \mathrm{M}$ TRIS buffer at $\mathrm{pH} 7.6$ for 15 minutes.

9 Apply swine anti-rabbit alkaline phosphatase complex (Sigma) for $\mathbf{4 5}$ minutes.

10 Wash in TRIS-saline for 10 minutes and add alkaline phosphatase substrate ${ }^{9}$ for 20 minutes to yield a red reaction product.

11 Counterstain in haematoxylin, wash in water, blue in Scott's tap water substitute and wash in water.

12 Mount in Glycergel (Dako).

Formalin fixed human tonsil provided positive controls for immunoglobulins in each case.

Table 2 Antibody identity and concentration used for immunofluorescence technique

\begin{tabular}{lll}
\hline & Manufacturer & Dilution \\
\hline Rabbit anti-human IgG & (Capell) & 1 in 15 \\
Rabbit anti-human IgA & (Wellome) & 1 in 4 \\
Rabbit anti-human IgM & (Wellcome) & Neat \\
Rabbit anti-human C1q & (Hoechst) & Neat \\
Rabbit anti-human C3 & (Hoechst) & 1 in 2 \\
Rabbit anti-fibrinogen & (Hoechst) & Neat \\
\hline
\end{tabular}

Negative controls were performed in all cases by omitting the primary antibody.

The protocol for the immunofluorescence technique was as follows:

1 Cut frozen sections $(7-10 \mu \mathrm{m})$, mount on glass slides, and air dry for 30 minutes.

2 Wash twice in phosphate buffered saline (PBS) for 10 minutes and fix in a 50:50 (v:v) mixture of ether and alcohol for 10 minutes.

3 Replace the ether alcohol mixture with $95 \%$ alcohol for 20 minutes and then wash the slides a further three times in PBS (five minutes for each wash).

4 Incubate the sections for 30 minutes with the fluorescein labelled primary rabbit antibodies listed in table 2 following preabsorption of these antibodies with mouse liver (Capell).

5 Wash three times in PBS and mount in Cytifluor (Cytifluor Ltd.); apply cover slips and examine under ultraviolet light.

One slide is treated with PBS alone so that any autofluorescence may be identified.

Analysis of the slides stained with IAP was undertaken by observers at Glasgow Royal Infirmary while immunofluorescence observations were made at the Department of Immunopathology, Western Infirmary, Glasgow.

In all but five of the cases material subjected to ultrastructural study provided an additional element of quality control.

\section{Results}

Of 80 renal biopsies performed, 62 were subjected to both immunofluorescence and IAP examination. These included 18 cases of IgA nephropathy, 11 of membranous nephropathy, seven of mesangioproliferative disease associated with IgM deposition, three cases of anti-glomerular basement membrane disease, three of lupus nephritis and three cases of post-infectious glomerulonephritis. Twelve cases were subjected to IAP treatment only when the biopsy material submitted for immunofluorescence yielded no glomeruli. In the six cases in which glomeruli were absent from the sample submitted for light microscopy, only immunofluorescence was possible.

In the 62 cases where both techniques were practicable the comparative results are listed in table 3. The overall concordance rate for the two methods for all antibodies was $85 \%$, though this rose to $93 \%$ when analysis was restricted to the identification of immunoglobulin deposition.

The IAP technique was easily performed, consistent, and permitted accurate intraglomerular localisation of complexed or insuded immune deposits (figs 1-5). Mesangial deposits (fig 1) could easily be differentiated from capillary loop complexes (fig 2), and in cases of membranous disease a subepithelial location could be readily inferred from the peripheral, coarse, interrupted pattern evident at high power (fig 2, inset). Insudation of immunoglobulin showed a characteristic pattern (fig 3), while subendothelial location 
Table 3 Positive and negative correlations with each technique for each antibody used

\begin{tabular}{llll}
\hline & $\begin{array}{l}\text { Immunofluorescence } \\
\text { positive } \\
\text { IAP positive }\end{array}$ & $\begin{array}{l}\text { Immunofluorescence } \\
\text { positive } \\
\text { IAP negative }\end{array}$ & $\begin{array}{l}\text { Immunofluorescence } \\
\text { negative } \\
\text { IAP positive }\end{array}$ \\
\hline IgG (granular) & 10 & 0 & 1 \\
IgG (linear) & 0 & 3 & 0 \\
IgM (granular) & 45 & 1 & 5 \\
IgA (granular) & 17 & 1 & 2 \\
C3 & 15 & 18 & 5 \\
Clq (granular) & 4 & 0 & 12 \\
Fibrinogen & 5 & 5 & 2 \\
\hline
\end{tabular}

of deposits was equally easily demonstrable (fig 4). The intensity of staining with each technique was similar for antibodies to IgA and IgM but anti-IgG staining by IAP was of slightly lower intensity when compared with immunofluorescence. The IgG antibody was also subject to a greater degree of slide variation and background staining and required more attention to accuracy of dilution, especially on changing to a new batch of primary antibody.

In certain specific areas, however, correlation was significantly lower. C3 staining by
Figures $1 a$ and $1 b$ Well defined mesangial deposits (arrows) are easily visible in this case of $\operatorname{Ig} A$ nephropathy. There is no evidence of peripheral loop deposition (arrowhead). (Anti-IgA by IAP method)

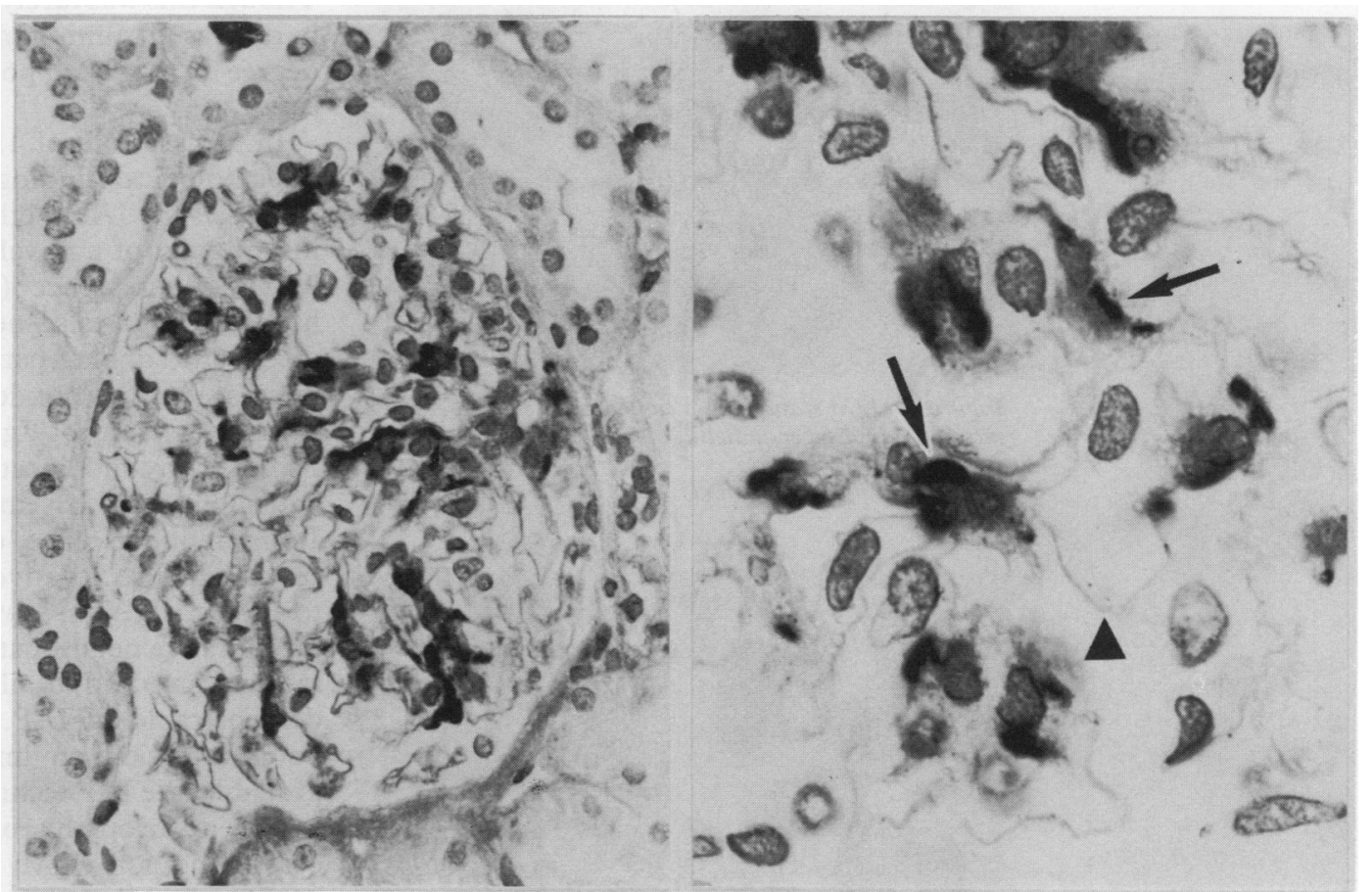

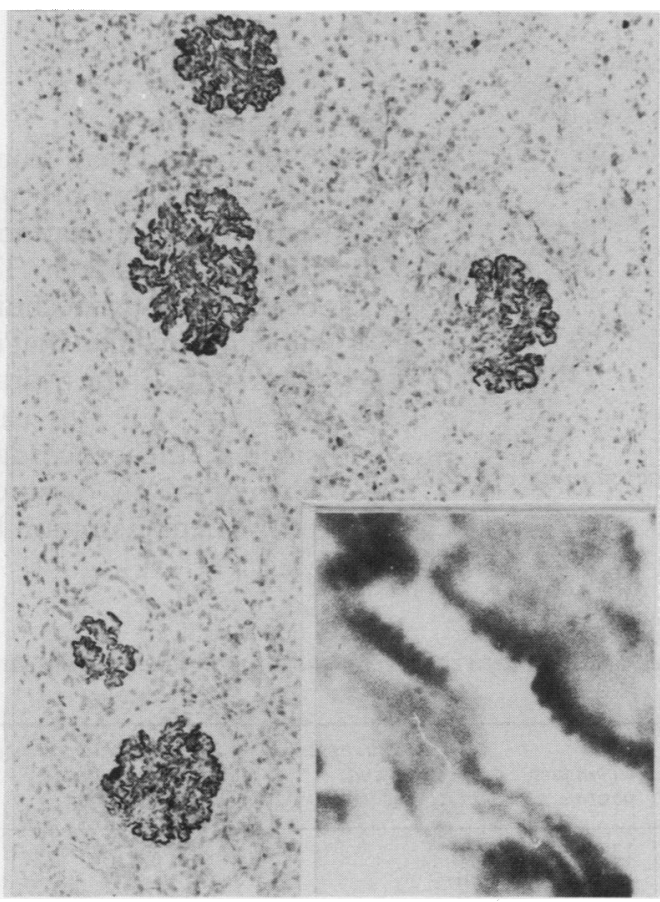

Figure 2 Granular loop deposits of IgG in a case of membranous nephropathy. The inset shows a subepithelial location. (Anti-IgG by IAP method)

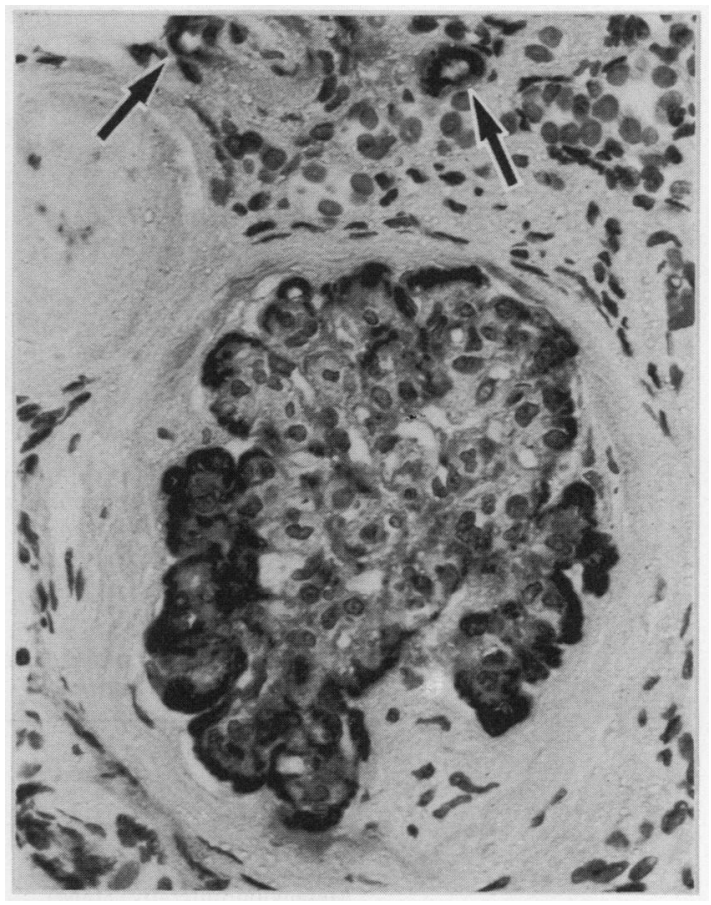

Figure 3 Glomerular capillary tuft and arteriolar (arrows) staining of insuded IgM in diabetic nephropathy.

(Anti-IgM by IAP method) 


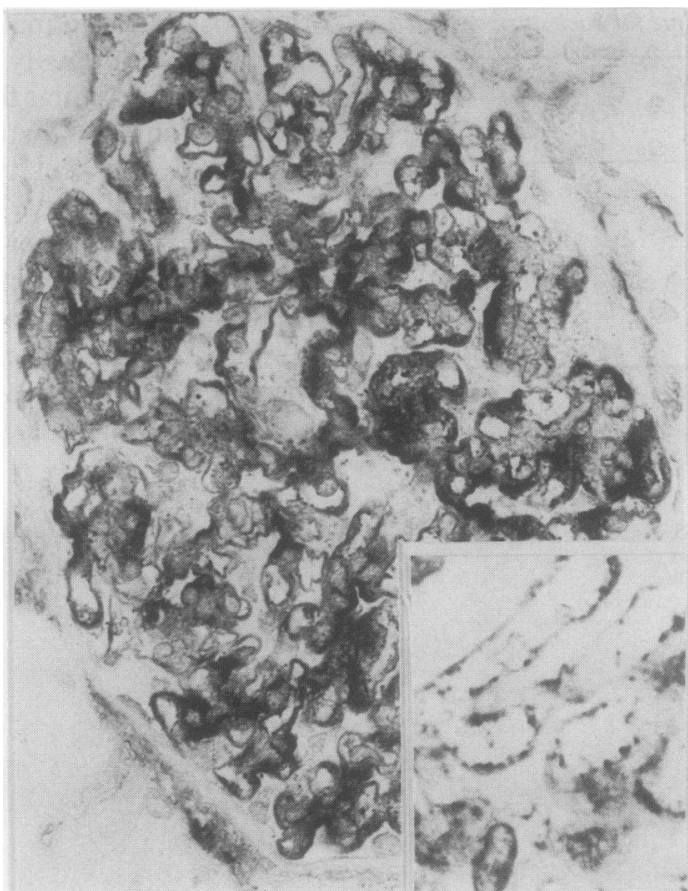

Figure 4 IgM staining in a case of SLE shows both mesangial and subendothelial (inset) deposition of immune complexes.

(Anti-IgM by IAP method)

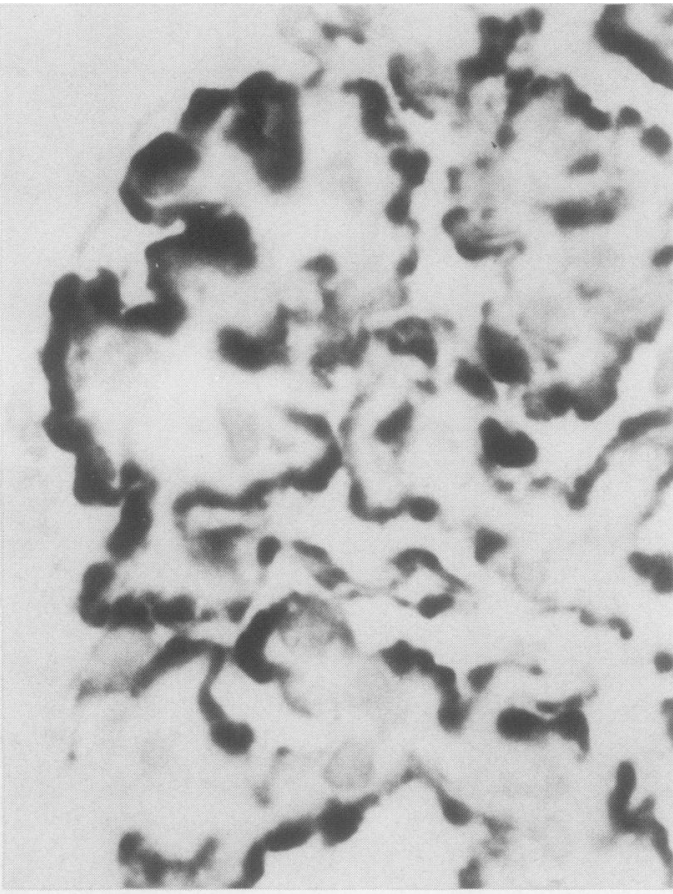

Figure 5 Granular capillary loop staining of C3 in a case of membranous nephropathy. (Anti-C3 by IAP method)
IAP (fig 5) was positive in less than $50 \%$ of immunofluorescence positive cases. A similar result was evident for fibrinogen, although in many of the immunofluorescence positive cases its pathological relevance was doubtful. $\mathrm{Clq}$ staining by IAP, while detected in all four of the cases positive on immunofluorescence, was characterised by what seemed to be an extremely high false positive rate.

In addition to glomerular staining, the immunoglobulin and C3 antibodies produced variable degrees of tubular (epithelial and cast) vascular, and plasma cell staining with IAP. Though this was rarely of diagnostic relevance, it did, as in immunofluorescence, provide a degree of internal positive control for such cases.

In 35 cases out of 41 where positive immunopathological findings were crucial to the diagnosis the IAP method provided diagnostic information of equivalent accuracy to that of immunofluorescence (table 4). There were, however, four false negative results where a diagnostic staining pattern noted on immunofluorescence was not detected by IAP. It failed to stain mesangial $\operatorname{IgA}$ in one case of $\operatorname{IgA}$ nephropathy and linear IgG in all three cases of anti-glomerular basement membrane disease. Immunofluorescence, on the other hand, also produced false negative results through failure to detect IgA in two cases of mesangioproliferative nephropathy where deposits were rather scanty and focally distributed, but easily demonstrable on IAP. In the 12 cases where no information on immunofluorescence was available, the IAP technique detected two cases of IgA nephropathy, one of membranous disease, one of IgM associated mesangioproliferative disease, and one of post-infectious glomerulonephritis, all five diagnoses being supported by electron microscopical findings.

\section{Discussion}

Our study differs from those previously published by being prospective and using a standardised short fixation time and alkaline phosphatase as marker. The overall concordance between the two techniques was $85 \%$, a slightly higher value than that in the previous studies. ${ }^{68}$ In practice, if the overall pattern rather than the absolute value for each test reagent is considered, the concordance rate rises to $90 \%$. Two of the six discordant results were related to the demonstration by the IAP technique of

Table 4 Positive and negative correlations with each technique for individual disease categories with diagnostic immunostaining patterns

\begin{tabular}{lllll}
\hline $\begin{array}{l}\text { Disease } \\
\text { category }\end{array}$ & $\begin{array}{l}\text { Both } \\
\text { techniques } \\
\text { positive }\end{array}$ & $\begin{array}{l}\text { Immunofluorescence } \\
\text { positive and } \\
\text { IAP negative }\end{array}$ & $\begin{array}{l}\text { Immunofluorescence } \\
\text { negative and } \\
\text { IAP positive }\end{array}$ & $\begin{array}{l}\text { No immunofluorescence } \\
\text { available } \\
\text { IAP positive }\end{array}$ \\
\hline $\begin{array}{l}\text { IgA nephropathy } \\
\text { Membranous }\end{array}$ & 13 & 1 & 2 & 2 \\
Mesangioproliferative (IgM) & 9 & 0 & 0 & 1 \\
Anti-glomerular basement & 7 & 0 & 0 & 1 \\
membrane disease & 0 & 3 & 0 & 0 \\
SLE & 3 & 0 & 0 & 0 \\
Post-infectious glomerulonephritis & 3 & 0 & 0 & 1
\end{tabular}


discrete focal, segmental, mesangial deposits of IgA in a substantial proportion, but by no means all of the glomeruli. Immunofluorescence, inevitably performed on a smaller sample, failed to show any immunoglobulin deposition. Electron microscopical examination in both cases showed discrete mesangial electron dense deposits. Conventional wisdom regards IgA nephropathy as invariably diffuse in its pattern of immunoglobulin deposition, but the clinical, light microscopical, and ultrastructural patterns observed in these cases were typical of mesangial IgA disease, and we believe, therefore, that given positive immunohistochemistry, albeit focal, they should be classified as such. It remains open to question whether the focal pattern of IAP and the negative immunofluorescence are simply a consequence of coincidental technical aberrations or whether these cases represent a variant form of IgA nephropathy. At least one previous case in our experience, however, seemed to show focal mesangial IgA staining on immunofluorescence, thus suggesting that the latter possibility is worthy of further investigation. If truly focal disease exists then it would seem that the larger sample provided by the IAP technique is likely to prove advantageous in its detection.

The value of the IAP technique was further confirmed by its provision of useful diagnostic information in five cases where no tissue was available for immunofluorescence examination.

Immunoalkaline phosphatase produced four false negative results. One case of mesangial IgA nephropathy was not detected. This, however, was probably due to a technical error such as omission of the primary antibody since, at a later date, restaining showed a diagnostic the IAP method was its constant failure to detect linear IgG immunopositivity despite the description of accurate detection by McIver et $a l .{ }^{67}$

Efforts to enhance the staining in the three cases of anti-glomerular basement membrane disease by varying duration of trypsinisation, incubation times, and primary antibody concentrations failed. Conditions which gave a positive result in test cases tended to produce high background and pseudolinear staining in negative controls. The reasons for failure are unclear, but the differences in the avidity of the primary antibodies used may have been relevant.

Staining for complement components during the study period gave variable results, correlation with immunofluorescence being much poorer than for immunoglobulin. In most cases, however, this did not present any diagnostic problem. Anti-C3, though specific, gave many false negative results. This may, in part, be due to trypsinisation which gives rise to partial C3 cleavage with a consequent loss of antigenic determinants. As we have recently been obtaining more consistent results with this antibody, however, we believe that antibody deterioration after prolonged storage and variation in the initial absorption and purifying procedure may also have compromised earlier tests. Staining with anti-Clq was characterised by a high false positive rate on IAP and seemed to produce a pattern of immunopositivity similar in distribution to that for anti-IgM. This obviously casts doubt on the specificity of this antibody for its antigen in fixed paraffin wax embedded material. The possible importance of this failure relates to the diagnosis of renal systemic lupus erythematosus (SLE) where the presence of glomerular C1q immunopositivity can help discriminate between that disease and other forms of glomerulopathy. In one particular case of SLE, however, C1q staining of loops and mesangium was of much greater intensity than that of the other antibodies of the panel, making a cross reaction much less likely. Striking Clq staining by IAP may therefore have genuine, if limited, diagnostic importance.

Although the IAP technique seems to be reliable and accurate, some interpretative experience is required before it yields consistent diagnostic results. Most importantly, a few biopsy specimens show positive staining of plasma components within capillaries of the glomerular tuft with most of the antibodies in the panel. Entrapment of the plasma within capillaries is less of a problem with immunofluorescence as the tissue sections receive multiple washes before fixation. Plasma derived staining on IAP, while clearly intravascular in most cases, can mimic subendothelial loop staining in others (fig 6). This spurious staining pattern is usually produced by all the antibodies, including fibrinogen, and tends to be associated with obvious intracapillary red blood cells and plasma on haematoxylin and eosin staining. If attention is paid to these

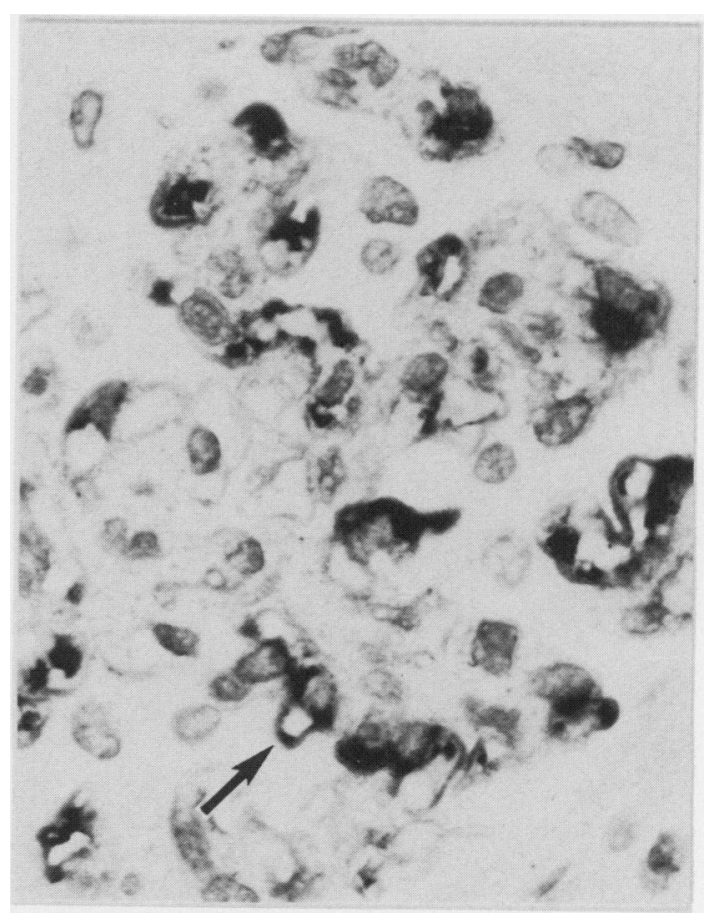

Figure 6 Intracapillary plasma staining mimicking subendothelial staining (arrow) in a case of minimal change disease.

(Anti-IgG by IAP method) 
details it can easily be differentiated from immunopositivity of diagnostic relevance.

At one point during the study period, due to a shortage of reagents, we were compelled to use horseradish peroxidase as a signalling system. Despite using an identical procedure to IAP, save for the inclusion of an endogenous peroxidase blocking step, we were unable to detect granular IgG in a case of membranous nephropathy. The subsequent repeat test, however, using the IAP system yielded a typical diagnostic pattern. This provided further support for our initial impression that alkaline phosphatase has greater sensitivity as a signalling system.

As the final red reaction product of the IAP technique is alcohol soluble, these slides must be mounted in an aqueous medium. Although this introduces a theoretical problem of drying out, we have not experienced this in the past two years using Dako Glycergel as mounting medium. A dark stippling artefact in the mounting medium close to positively staining areas may develop after prolonged storage. This may render satisfactory photography difficult, although the tissue pattern remains adequate for late diagnostic review. We are currently exploring modifications to the technique to improve further the permanence of preparations.

Since the completion of the study we have continued to use the IAP technique on a routine basis and remain convinced of its value. We feel, however, that it should be emphasised that the acquisition of interpretative skills depends on the ready availability of an immunofluorescence bench mark, backed up by electron microscopy. Moreover, the need for an immunofluorescence service is likely to be sustained in academic departments, particularly if there is increasing necessity to detect antigens vulnerable to formalin fixation.

In conclusion, we believe that the IAP technique, applied to paraffin wax sections from biopsy material subjected to a uniform short fixation time, is better suited to nephropathological diagnosis than previously described immunoperoxidase techniques and that it could be used as a satisfactory alternative to conventional immunofluorescence except where anti-glomerular basement membrane disease is suspected.

1 Lange K, Treser G, Sagel I, Ty A, Wasserman E. Routine immunohistology in renal diseases. Ann Intern Med 1966;64:25-40.

2 Turner DR, Wilson DM, Lake A, Heaton JM, Leibowitz S, Cameron JS. An evaluation of the immunoperoxidase technique and renal biopsy diagnosis. Clin Nephrol 1979;11:13-17.

3 Davey FR, Busch JG. Immunohistochemistry of glomerulonephritis using horseradish peroxidase and fluorescein-labelled antibody: A comparison of two technics. Am J Clin Pathol 1970;53:531-6.

4 Tubbs RT, Gephardt G, Valenzuela R, Deodhar S. An approach to immunomicroscopy of renal disease with immunoperoxidase and periodic acid-Schiff counterstain (IMPAS stain). Am J Clin Pathol 1980;73:240-4.

5 Curran RC, Gregory J. Effects of fixation and processing on immunohistochemical demonstration of immunoglobulin in paraffin sections of tonsil and bone marrow. J Clin Pathol 1980;33:1047-57.

6 MacIver AG, Giddings J, Mepham BL. Demonstration of extracellular immunoproteins in formalin-fixed renal biopsy specimens. Kidney Int 1979;16:632-6.

7 MacIver AG, Mepham BL. Immunoperoxidase techniques in human renal biopsy. Histopathol 1982;6:249-67.

8 Sinclair RA, Burns J, Dunnill MS. Immunoperoxidase staining of formalin-fixed paraffin-embedded, human renal biopsies with a comparison of the peroxidaserenal biopsies with a comparison of the peroxidaseantiperoxidase (PAI

9 Ponder BA, Wilkinson MM. Inhibition of endogenous tissue alkaline phosphatase with the use of alkaline phosphatase conjugates in immunohistochemistry. J Histochem Cytochem 1981;29:981-4. 